\title{
Our evolving climate: communicating the effects of climate variability
}

\author{
Ed Hawkins \\ NCAS-Climate \& Walker Institute, University of Reading \\ e.hawkins@reading.ac.uk
}

Accepted article for Weather - 29th November 2010

\begin{abstract}
Our evolving climate is influenced by several factors such as greenhouse gases and solar output, coupled with the inherent variability of the climate system. Natural fluctuations in climate can temporarily mask or enhance the long-term upwards trend in temperatures, and contribute to uncertainty in climate projections, especially on regional scales. Understanding and acknowledging these variations is important for society and policymakers. This article aims to communicate the impacts of natural variability and discuss recent progress in understanding and predicting natural climate fluctuations. This progress offers potential to narrow uncertainty in climate predictions and may aid efficient adaptation to our changing climate.
\end{abstract}

It is "very likely" that humans have caused most of the warming of the Earth's climate since the mid-20th century; this was a key conclusion of the 4th Assessment Report (AR4; Solomon et al., 2007) of the Intergovernmental Panel on Climate Change (IPCC). For the UK, 2006 was the warmest year recorded to date, however 1998 remains the warmest year on record for the global average ${ }^{1}$. This has led many to question whether the climate is changing as projected by the IPCC.

This article aims to portray and communicate the important role played by natural variability in our evolving climate. Understanding and acknowledging these variations is important for society and policymakers. Much of this variability is chaotic and unpredictable but some significant fraction is potentially predictable, providing an opportunity to narrow the uncertainty in climate predictions of the coming decade.

\section{Our evolving climate}

The Earth's climate is changing primarily due to two factors - (i) the response to external forcings such as greenhouse gases, solar output and volcanoes, coupled with, (ii) the inherent internal (or natural) variability of the climate system. These natural fluctuations in climate can temporarily mask or enhance any long-term trends to the extent that one year, or even decade, will not necessarily be warmer (or wetter/drier) than the last.

On seasonal and annual timescales, the main climate influence on global scales is the El Nino Southern Oscillation (ENSO) phenomenon - the periodic warming and cooling in the Pacific

\footnotetext{
${ }^{1}$ According to the HadCRUT3 global temperatures dataset used by the IPCC (Brohan et al., 2006; also see Fig. 5 later), although other datasets find more recent years to be warmer than 1998 .
} 
Ocean. It was the large El Nino in 1998 which helped produce the observed record global temperatures. Although ENSO has some impact in Europe (Ineson and Scaife, 2009), especially in winter, it does not dominate climate variability for the UK. Importantly, there are also longer decadal variations in climate, usually linked to changes in the ocean circulation. This type of natural decadal variability can make it difficult to distinguish between a long-term tend and a short-term natural fluctuation, illustrated for European (EU) temperatures in Fig. 1. The black lines in each panel show observed annual average EU temperatures from 1950 onwards. Temperatures have varied considerably from year-to-year, and decade-to-decade during this period of observations.

Various global climate models (GCMs), designed by different research centres around the world, predict climate assuming high, medium or low 'scenarios' of future greenhouse gas emissions. These GCMs all differ somewhat in their response to emissions, giving a range of 'projections' of how the climate will evolve through the coming century. For instance, the range of GCM responses for EU temperatures in the low emissions scenario indicate an increase in temperature between 1.5-3.5K (Fig. 1a, blue lines), i.e. there is some uncertainty in the magnitude of temperature change even when the same future emissions are assumed in each GCM projection. It is also clear that both the historical observations and temperature projections exhibit variability. However, when presenting this range of responses, the IPCC tends to show the average and spread of the GCM projections, indicating a relatively smooth increase in temperatures over the coming century (Fig. 1b, blue shading). Although this representation provides a range for the likely increase in future temperatures, it tends to disguise the natural variability of climate. One particular projection illustrating the impact of internal climate variability on EU temperatures is shown in Fig. 1c. To highlight the importance of the natural fluctuations in climate, a decade which shows a sharp decline in temperatures is then chosen from this particular projection (Fig. 1d), demonstrating how a climate trend may be misrepresented when considering a relatively short time period. Although this particular decade is chosen specifically, it is not unusual - there are also several periods of rapid warming and rapid cooling in the observational record.

So, what is the chance of one year being cooler than the last? For global mean temperatures in the future, there is roughly a $40 \%$ chance that one year will be cooler than the last (Fig. 2a). Equivalently, this could be expressed as ' 2 in every 5' years. For smaller regions, such as Europe or the UK, this chance is higher (around 47\%). Although this may seem quite counter-intuitive, there is not much difference between the chances of a head or tails when tossing a coin, and whether the temperature in Europe one year will be warmer or cooler than the last.

However, for longer timescales the odds change because of the gradual upwards trend in temperatures. Decades which exhibit a cooling (or a negative temperature trend) are only expected occasionally in the future for the global mean (about $5 \%$ of decades), but these chances increase to $24 \%$ of future decades for Europe and $36 \%$ of future decades for the UK (Fig. 2b). Expressed as odds, there is roughly a 1-in-3 chance of a particular future decade exhibiting a cooling trend for the UK. This is a key point which is essential for society and policymakers to appreciate temperatures are expected to (temporarily) go down as well as up, even in a warming climate.

Another interesting question to ask is, how long might we have to wait before a warmer year occurs? Although, we may be surprised that 1998 is still the warmest year recorded, the GCMs suggest that, for global mean temperature, it is possible that we could wait 17 years; and so far we have been waiting 12 years. For smaller regions, climate fluctuations are larger and for UK 
temperatures, we could wait nearly 50 years, although usually it would be under 5 years.

Although there are caveats to this simple analysis, it demonstrates how climate variability is likely to affect an individual's interpretation of whether there are long term changes in climate or not. To determine whether the climate is truly changing, it is essential to consider long (multidecadal) timescales and large spatial scales.

\section{DEMONSTRATING CLIMATE VARIABILITY AT HOME}

Take a shuffled pack of playing cards, with red cards representing 'warm' years and black cards 'cool' years. When dealing the pack there will be times when several warm or cool years appear together. Next, remove some black cards from the pack, and reshuffle. This pack now represents a changed 'climate' with less cool years. When dealing the pack for a second time, there will be more periods of warm years, but probably periods of cool years as well. Even though the climate has warmed, every year need not be hot!

\section{Variability as an analogue for the future}

As described above, our climate is changing relatively slowly compared to human memory. As significant climate changes tend to only appear after many years, it can be hard to appreciate how the climate has already changed, and hard to imagine the impacts that are projected to occur; the climate in the 2050s may seem quite a remote prospect. However, natural climate fluctuations can help us appreciate what living in a changed climate would be like by acting as an analogue for what the future holds.

For example, the extreme European summer heatwave of 2003 gave us a taste of what is projected for Europe in the 2050s. The heatwave probably caused an estimated additional 30,000 deaths across Europe, an increase in forest fires, lower crop yields and disrupted transport (UNEP Environment Alert Bulletin, 2004). Although climate change did not cause the heatwave, it has made such events more likely. In fact, Stott et al. (2004) suggested that the chances of such an extreme heatwave had been at least doubled to about 1-in-every-250 years by the climate change that has already happened, and that this risk is increasing rapidly.

The extreme summer temperatures in 2003 are evident in the observations (Fig. 3, black line). Projections of summer EU temperatures (for 'medium' future emissions) indicate that the type of summer experienced in 2003 will become normal (i.e. 1-in-every-2 years) by around 2050, and that Europe would rarely experience a summer as cold as 2003 by the end of the 21st century (Fig. 3, blue shading).

\section{Variability as uncertainty}

Although climate scientists are confident in the direction of any long term change in temperatures, there is a range of estimates for the magnitude of the change, i.e. some uncertainty. However, there are several different sources of uncertainty in our projections of climate. As indicated previously, the climate we will experience in the coming decades is significantly influenced by natural fluctuations, giving rise to some uncertainty over the trajectory the climate will follow (the internal variability component of uncertainty). There is also uncertainty in future climate due to different GCMs having different responses to greenhouse gases etc (termed model response uncertainty, for example, as shown by the spread in projections in Fig. 1a), and also uncertainty 
in the rate of future greenhouse gas emissions (termed scenario uncertainty). The potential to narrow uncertainty in climate projections depends on which source of uncertainty is dominant. For example, climate science can tackle the model response uncertainty, but can do little to reduce the uncertainty in future emissions because this depends on economic development and human behaviour.

To determine the dominant source of uncertainty, we can examine how the relative magnitudes of the different sources of uncertainty vary for different regions over the 21st century (Hawkins and Sutton, 2009). For example, projections of UK temperatures at the end of the 21st century vary from 1-3K above recent levels ${ }^{2}$. This total uncertainty in the projections can be separated into the three sources of uncertainty (Fig. 4). The natural, internal variability component (orange) is the largest source of uncertainty for the next couple of decades, and the choice of emissions scenario (green) is relatively unimportant for the near-term. This may be surprising, but it takes around 30 years for any changes in emissions to have an appreciable effect on the climate (so called 'climate inertia'), suggesting that we have little chance of avoiding climate change in the UK over the next few decades and we may have to adapt. Towards the end of the century, the particular levels of greenhouse gas emissions have a larger impact on temperatures. Thus, we are committed to further increases in temperature, and any actions taken now to change our emissions will only have an influence later in the century. However, waiting to reduce emissions will further delay the climate response and lead to a larger increase in temperatures. The remaining uncertainty is due to our choice of climate model (blue). Reducing this uncertainty by improving our climate models is possible, but challenging, and is the only way to narrow uncertainty in long term projections.

\section{Predicting a decade ahead}

Climate projections assessed by the IPCC are not true 'predictions' per se; they attempt to forecast the trends in climate (Fig. 1b); variability seen around the trends are examples of how the climate might evolve (Fig. 1c). For considering mitigation of climate change, the magnitude of the upwards temperature trend is important, as this dictates how quickly greenhouse gas emissions need to decrease to meet targets for global mean temperatures. The IPCC present their climate projections as depicted in Fig. 1b for this reason.

However, it is important to make planning decisions so society can adapt to a changing climate on decadal timescales (e.g. Sutton, 2005; Wilby and Dessai, 2010). For example, an assessment of when to replace railway track might include the risks of a heatwave causing the lines to buckle, as happened in the UK during the heatwave of 2003. For most regions, it is natural variability which dominates this decadal timescale, as seen above for UK temperatures.

As a further example, imagine we are in the year 2020 and the climate evolves along the blue line in Fig. 1c. The impacts of a decrease in temperatures such as that seen in the 2020s in this hypothetical case, would be significant, e.g. to farmers planning which crops to plant. It may even cause renewed doubts over whether humans are causing the climate to change. After seeing a dramatic increase in temperatures over the previous decade, could we predict the rapid decline that subsequently happens?

We do not yet know the answer to this key question. Some fraction of these fluctuations are

\footnotetext{
${ }^{2}$ Also see the latest UK Climate Projections (http://ukclimateprojections.defra.gov.uk) which describe in detail the range of possible future climates of the UK.
} 
chaotic, and inherently unpredictable, but some derive from the slower evolution of the climate system. Research into the causes of such decadal variability focusses on the role of the oceans, and particularly for Europe, the thermohaline circulation in the Atlantic Ocean (e.g. Sutton and Hodson, 2005), the role of the stratosphere (e.g. Scaife et al., 2005) and the climate response to the solar cycle (e.g. Lockwood et al., 2010). A more comprehensive understanding of the causes of such variability may also allow testing of climate models, and to help distinguish variability from long-term trends.

In particular, if we can predict the evolution of ocean temperatures (e.g. Smith et al., 2007) we may be able to predict some of the related impacts over land. For example, the Sahel region of Africa experiences significantly more rainfall when the tropical North Atlantic ocean surface temperatures are warm, and vice versa (e.g. Folland et al., 1986). In fact, for many tropical regions, the projected trends in precipitation are relatively small compared to the magnitude of natural variability. For these regions, the focus should remain on adapting to the impacts of precipitation variability.

The IPCC do not currently assess predictions of the exact trajectory of the climate, as this does not impact the key issue of mitigating against the expected change in climate by the end of the century. However, the next IPCC report will be different, and will include a multi-GCM comparison of near-term forecasts which will attempt to predict both the continuing climate response to greenhouse gases, and the internal variability component over the next decade (see Meehl et al., 2009).

This type of prediction is technically extremely challenging. To predict how the ocean will evolve requires accurate knowledge of the ocean state, especially below the surface, which in turn requires continuous monitoring of the global oceans. The quantity of ocean observations decreases going back in time, particularly below the surface. This limitation of data only allows a reasonable estimate of the ocean state to be derived from around 1960 onwards. The recent deployment of a network of around 3,000 autonomous ocean floats $\left(\mathrm{ARGO}^{3}\right)$ which circulate in ocean currents has substantially aided this endeavour. These floats take regular measurements of ocean temperature and salinity from the surface to a depth of around $2 \mathrm{~km}$, with the data transferred to monitoring stations via satellite.

Smith et al. (2007) produced the first climate predictions which compared forecasts with and without ocean state information at the start of the forecast. Examples of such decadal retrospective predictions of global temperatures are shown in Fig. 5. The blue shaded areas indicate the predictions made using the additional ocean data ('initialised') and the red lines are standard climate projections which did not use any extra ocean state information. Although both methods predict the upwards trend in temperatures since 1975, the initialised predictions predict the evolution of the climate more accurately, especially for the first year or two, demonstrating their potential to improve predictions and narrow uncertainty.

The inset in Fig. 5 shows individual forecasts started in 2005. Although the subsequent observations are generally within the range of predicted temperatures, they are at the lower end. This could have happened for at least three possible reasons, (i) by chance, or (ii) because the model is not able to fully predict the natural variability (such as the 2008 La Nina), or (iii) because there are other external forcings which are not yet fully accounted for in the model.

Numerical weather prediction has benefited from continually assessing the ability of the com-

\footnotetext{
${ }^{3}$ http://www.argo.ucsd.edu/
} 
puter models to make forecasts. Further testing of our climate models in a similar way is vital to increase confidence in their use for longer term projections and potentially identify parts of the model which require improvements. Whether these types of decadal prediction can produce more accurate forecasts for smaller regions, and of other relevant climate variables, such as precipitation, remains to be seen (although see Smith et al., 2010). The provision of climate information relevant for adaptation - or 'climate services' - is growing because of this potential for more accurate near-term predictions.

\section{Summary}

Our climate is evolving. Although global and regional temperatures generally have a long-term upwards trend, the presence of natural variability means that each year, or decade, is not necessarily warmer than the last. Communication of the impact of natural fluctuations is vital for decision makers and for a sceptical public. Progress in understanding and predicting the natural fluctuations in climate offers the potential to test and improve climate models, narrow the uncertainty in climate predictions and aid adaptation to our evolving climate. Meeting these substantial scientific challenges requires continued investment in global observations, more advanced climate models and better ways of testing climate models against observations.

\section{Author's note}

The author welcomes feedback on communicating climate variability and climate uncertainty. The figures included here are available from the author's website

(http://www.met.reading.ac.uk/ ed/blog/our-evolving-climate).

\section{Acknowledgements}

EH is supported by NCAS-Climate and the EU THOR programme. Thanks to Jon Robson for useful suggestions and for providing data, and to Sarah Keeley, Rowan Sutton, Sam Burgess and two anonymous reviewers for helpful comments on an earlier draft. The DePreSys PPE data was produced by Doug Smith (UK Met Office).

\section{References}

Brohan, P., J. J. Kennedy, I. Harris, S. F. B. Tett, and P. Jones (2006), Uncertainty estimates in regional and global observed temperature changes: a new dataset from 1850, J. Geophys. Res., 111, D12106, doi:10.1029/2005JD006548.

Easterling, D. R., and M. F. Wehner (2009), Is the climate warming or cooling?, Geophys. Res. Lett., 36, L08,706, doi:10.1029/2009GL037810.

Folland, C. K., T. N. Palmer, and D. E. Parker (1986), Sahel rainfall and worldwide sea temperatures, 1901-85, Nature, 320, 602-607, doi:10.1038/320602a0.

Hawkins, E., and R. Sutton (2009), The potential to narrow uncertainty in regional climate predictions, Bull. Amer. Met. Soc., 90, 1095-1107, doi:10.1175/2009BAMS2607.1. 
Ineson, S., and A. A. Scaife (2009), The role of the stratosphere in the European climate response to El Niño, Nat. Geosci., 2, 32-36, doi:10.1038/ngeo381.

Lockwood, M., R. G. Harrison, T. Woollings, and S. K. Solanki (2010), Are cold winters in Europe associated with low solar activity?, Environ. Res. Lett., 5, 024,001, doi:10.1088/1748$9326 / 5 / 2 / 024001$.

Meehl, G. A., et al. (2009), Decadal prediction: can it be skillful?, Bull. Amer. Met. Soc., 90, 1467-1485, doi:10.1175/2009BAMS2607.1.

Scaife, A. A., J. R. Knight, G. K. Vallis, and C. K. Folland (2005), A stratospheric influence on the winter NAO and North Atlantic surface climate, Geophys. Res. Lett., 32, L18,715, doi: 10.1029/2005GL023226.

Smith, D. M., S. Cusack, A. W. Colman, C. K. Folland, G. R. Harris, and J. M. Murphy (2007), Improved surface temperature prediction for the coming decade from a global climate model, Science, 317, 796-799, doi:10.1126/science.1139540.

Smith, D. M., R. Eade, N. J. Dunstone, D. Fereday, J. M. Murphy, H. Pohlmann, and A. Scaife (2010), Skilful multi-year predictions of Atlantic hurricane frequency, Nature Geosci., in press, doi:10.1038/ngeo1004.

Solomon, S., D. Qin, M. Manning, Z. Chen, M. Marquis, K. Averyt, M. M. B. Tignor, and H. L. Miller (Eds.) (2007), Climate Change 200\%: The Physical Science Basis. Contribution of Working Group I to the Fourth Assessment Report of the Intergovernmental Panel on Climate Change, Cambridge University Press, Cambridge, UK.

Stott, P. A., D. A. Stone, and M. R. Allen (2004), Human contribution to the European heatwave of 2003, Nature, 432, 610-614, doi:10.1038/nature03089.

Sutton, R. (2005), Informing adaptation: new challenges for the climate modelling community, Weather, 60, 186-189, doi:10.1256/wea.224.04.

Sutton, R. T., and D. L. R. Hodson (2005), Atlantic Ocean forcing of North American and European summer climate, Science, 309, 115-118, doi:10.1126/science.1109496.

UNEP Environment Alert Bulletin (2004), Impacts of summer 2003 heat wave in Europe, United Nations Environment Programme. Available from: http://www.grid.unep.ch/product/publication/EABs.php.

Wilby, R. L., and S. Dessai (2010), Robust adaptation to climate change, Weather, 65, 180-185, doi:10.1002/wea.543. 

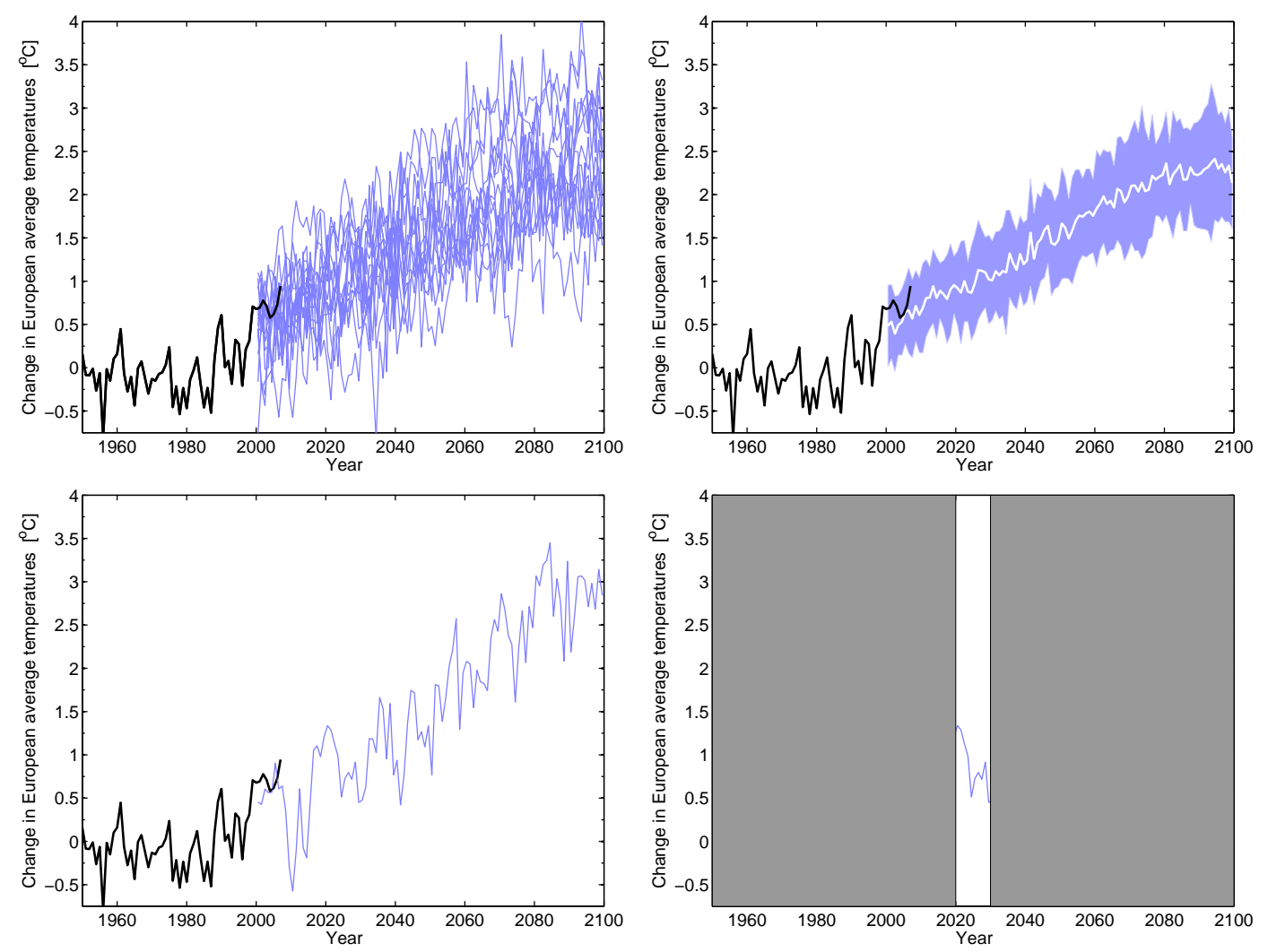

Figure 1: Illustrating natural climate variability in European (EU) temperatures. Observed EU temperatures from 1950 onwards (black; derived from Brohan et al., 2006) and projections for the 21st century from a range of global climate models (GCMs) using the 'low' future emissions scenario (SRES B2). (a) The individual projections from each GCM. (b) The mean (white) and spread (blue shaded) of the individual projections. (c) One particular projection for the 21st century. (d) One particular decade in the same projection. 
(a)
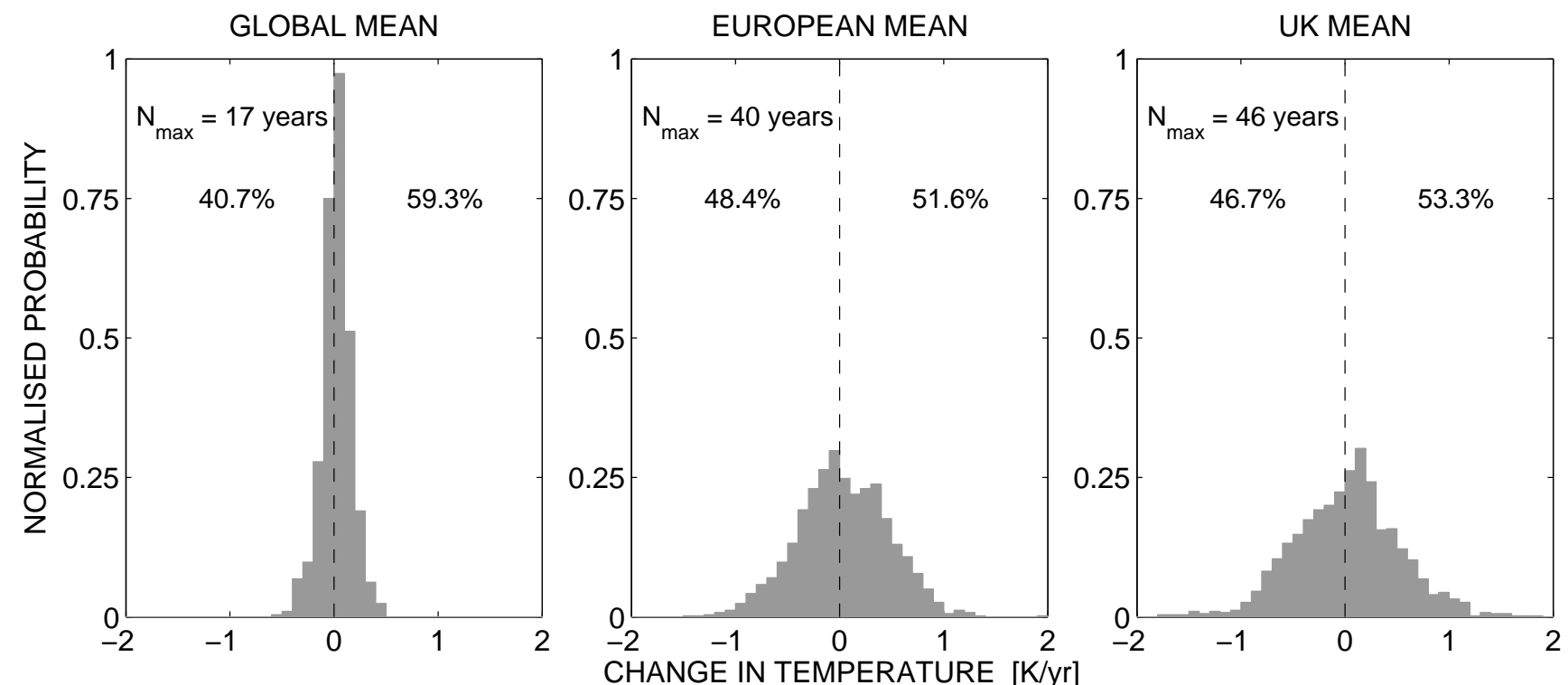

(b)
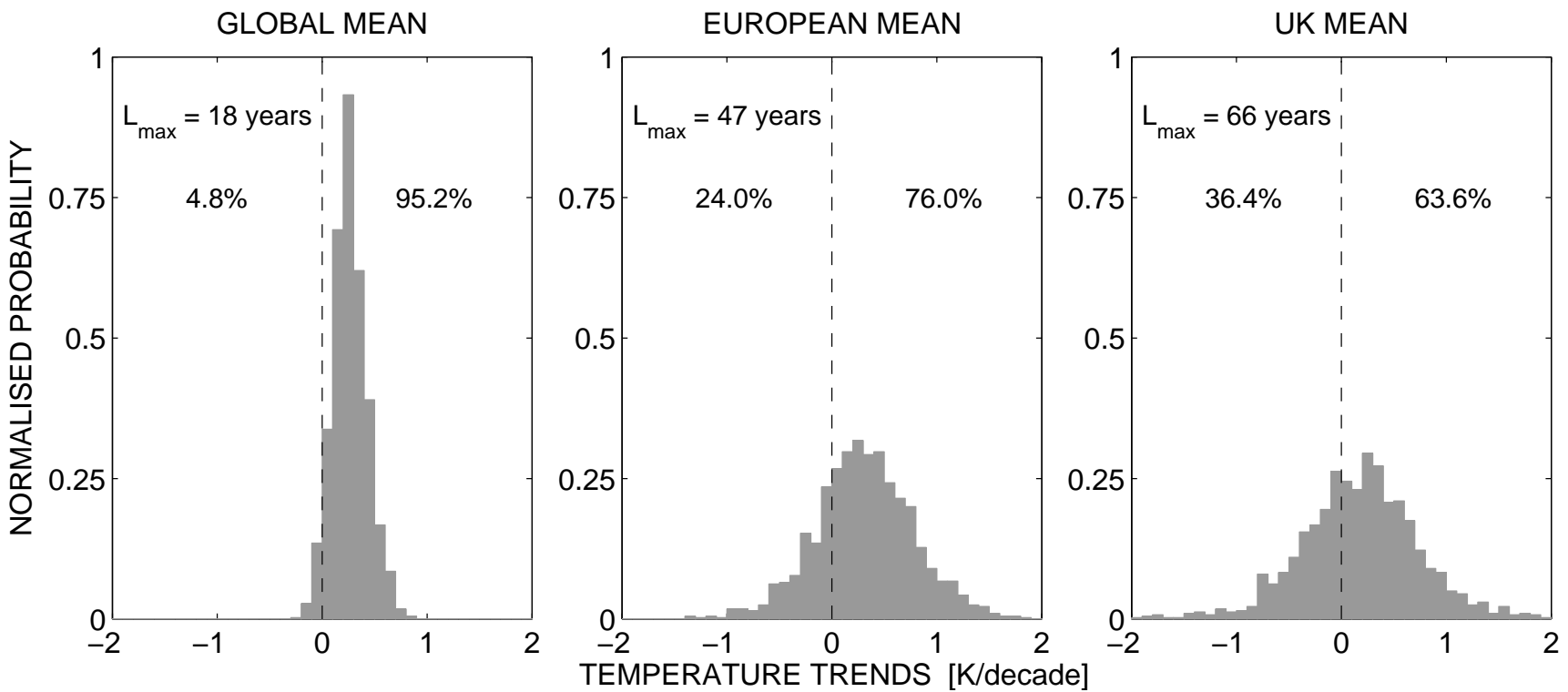

Figure 2: Histograms showing the (a) probability of a particular future temperature change between one year and the next, and (b) probability of a future decade with a particular temperature trend, for different regions as labelled. For smaller regions the chances of a decade with a negative temperature trend increases. Percentages shown are for differences and trends greater or less than zero. $\mathrm{N}_{\max }$ is the maximum number of years to wait before a warmer year occurs. $\mathrm{L}_{\max }$ is the longest period which may have a negative temperature trend. Results shown for IPCC 'medium' future emissions (SRES A1B) scenario. Note that these estimates do not include the impact of volcanic eruptions which would increase the chances of a negative decadal trend. Easterling and Wehner (2009) showed similar results for the global mean for decadal changes. 


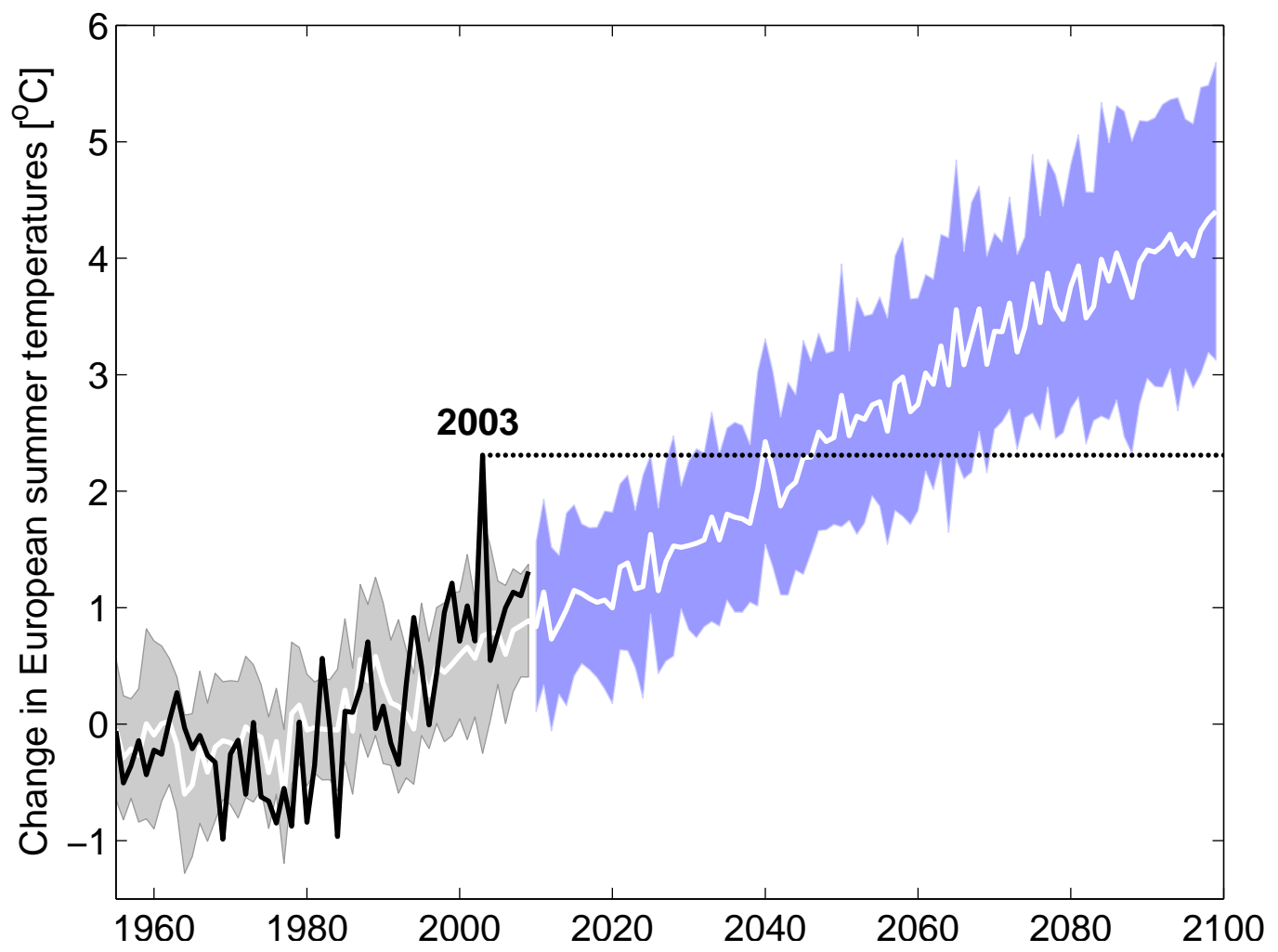

Figure 3: Observed EU summer temperatures (black; derived from Brohan et al., 2006), and IPCC AR4 projections of future summer temperatures using the 'medium' future emission scenario (SRES A1B, blue shading). The grey shading shows the spread in IPCC AR4 climate models when using historical external forcings. The dashed black line indicates the level of the 2003 summer. A similar figure using a single climate model was shown in Stott et al. (2004). 


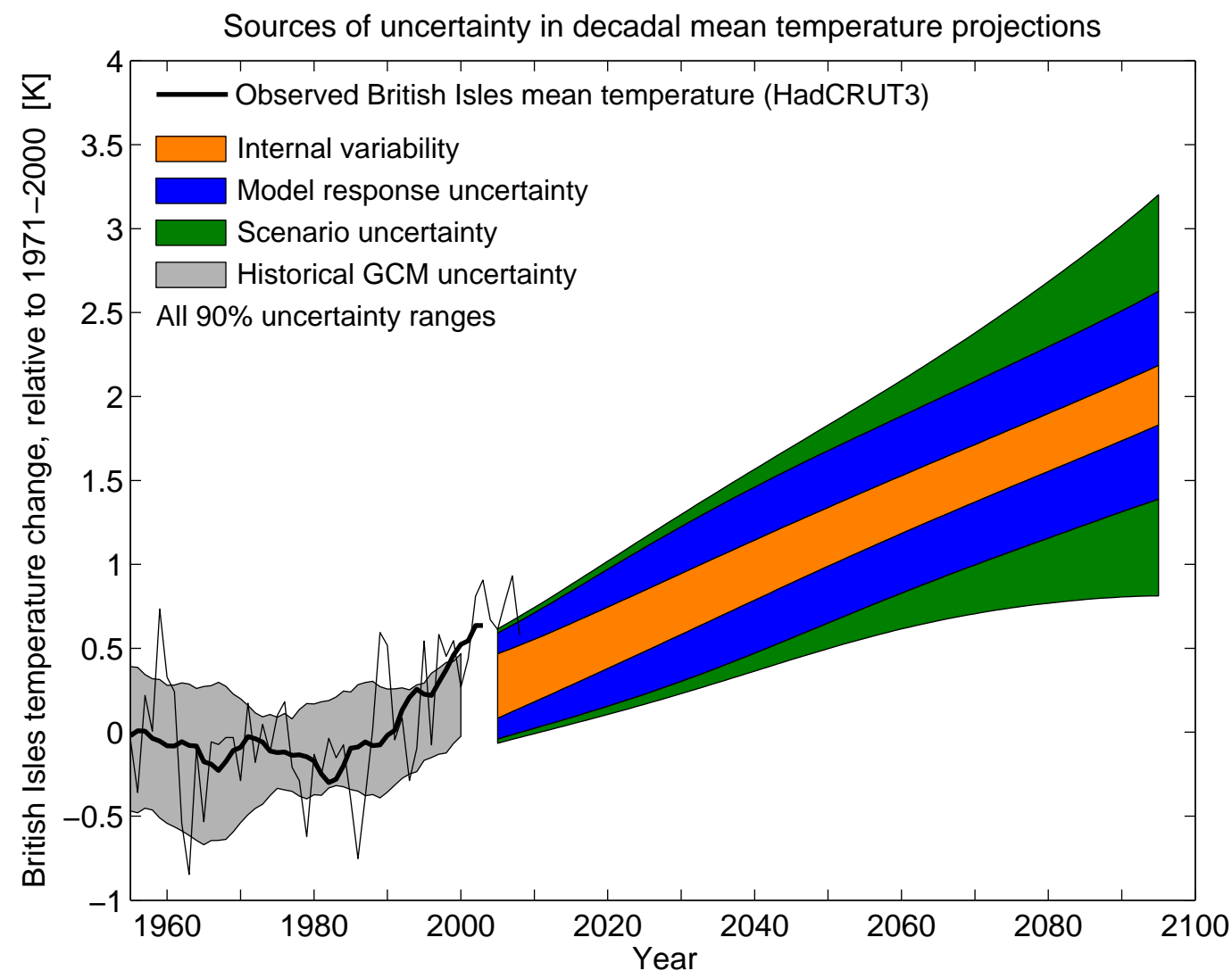

Figure 4: The relative importance of three sources of uncertainty in future UK temperature projections as coloured. The black lines show observed temperatures (thin - annual averages, thick - decadal averages; derived from Brohan et al., 2006). After Hawkins and Sutton (2009). 


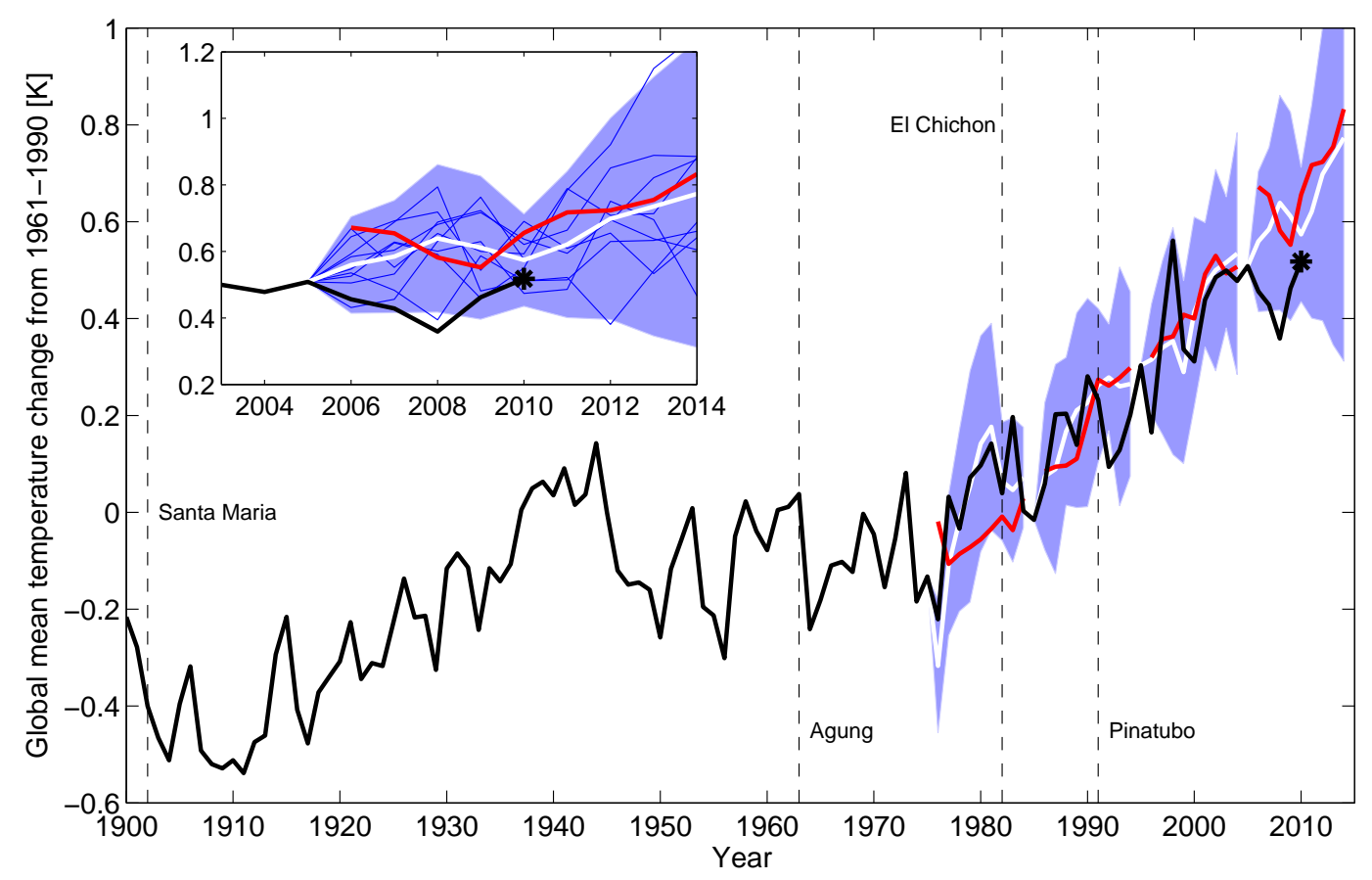

Figure 5: Example decadal predictions of global mean temperature started in 1975, 1985, 1995 and 2005 (blue shading) and the comparable projections which do not use the additional ocean state information (red lines), all taken from the Met Office Decadal Prediction System (DePreSys PPE; after Smith et al., 2007). The observations are shown in black (derived from Brohan et al., 2006), with provisional data for 2010 shown as the star. Although 1998 is the warmest year in the observations, the overall trend is upwards, with superimposed natural variability. The inset shows that the observations since 2005 are within, but at the lower end, of the expected range of predictions (blue lines), apart from 2008, which was a strong La Nina year. The labelled dashed lines indicate the major volcanic eruptions of the 20th century which tend to cool the climate and are unpredictable. 